\title{
Inactivation of microbial infectiousness by silver nanoparticles-coated condom: a new approach to inhibit HIV- and HSV-transmitted infection
}

This article was published in the following Dove Press journal:

International Journal of Nanomedicine

24 September 2012

Number of times this article has been viewed

\author{
A Mohammed Fayaz ${ }^{1, *}$ \\ Zhujun Aol,3,* \\ Morkattu Girilal ${ }^{2}$ \\ Liyu Chen ${ }^{3,4}$ \\ Xianzhong $\mathrm{XiaO}^{4}$ \\ PT Kalaichelvan² \\ Xiaojian Yao ${ }^{1,3}$
}

'Laboratory of Molecular Human Retrovirology, Department of Medical Microbiology, Faculty of Medicine, University of Manitoba, Winnipeg, MB, Canada; ${ }^{2}$ CAS in Botany, University of Madras, Guindy Campus, Chennai, Tamil Nadu, India; ${ }^{3}$ Department of Microbiology, ${ }^{4}$ School of Basic Medical Sciences, Central South University, Changsha, Hunan, People's Republic of China

*Both authors contributed equally to this work
Correspondence: Xiaojian Yao

Tel +l 2049775677

Fax + I 2047893926

Email yao2@cc.umanitoba.ca
Abstract: Recent research suggests that today's condoms are only $85 \%$ effective in preventing human immunodeficiency virus (HIV) and other sexually transmitted diseases. In response, there has been a push to develop more effective ways of decreasing the spread of the disease. The new nanotechnology-based condom holds the promise of being more potent than the first-generation products. The preliminary goal of this study was to develop a silver nanoparticles (Ag-NPs)coated polyurethane condom (PUC) and to investigate its antimicrobial potential including the inactivation of HIV and herpes simplex virus (HSV) infectiousness. The Ag-NPs-coated PUC was characterized by using ultraviolet-visible spectrophotometry, Fourier transform-infrared spectroscopy, high-resolution scanning electron microscopy, and energy-dispersive analysis of X-ray spectroscopy. Nanoparticles were stable on the PUC and not washed away by water. Morphology of the PUC was retained after coating. The NP binding is due to its interaction with the nitrogen atom of the PUC. No significant toxic effects was observed when human HeLa cells, 293T and C8166 T cells were contacted to Ag-NPs-coated PUC for three hours. Interestingly, our results demonstrated that the contact of the Ag-NPs-coated PUC with HIV-1 and HSV-1/2 was able to efficiently inactivate their infectiousness. In an attempt to elucidate the antiviral action of the Ag-NPs, we have demonstrated that the anti-HIV activity was primarily mediated by the Ag-NPs, which are associated with the PUC. In addition, the data showed that both macrophage (M)-tropic and T lymphocyte (T)-tropic strains of HIV-1 were highly sensitive to the Ag-NPs-coated PUC. Furthermore, we also showed that the Ag-NPs-coated PUC was able to inhibit the growth of bacteria and fungi. These results demonstrated that the Ag-NPs-coated PUC is able to directly inactivate the microbe's infectious ability and provides another defense line against these sexually transmitted microbial infections.

Keywords: silver nanoparticles, condom, HIV-1, HSV-1/2, antimicrobial

\section{Introduction}

Human immunodeficiency virus type 1 (HIV-1) and other sexually transmitted infections (STIs) are major health problems worldwide. The World Health Organization estimates that 340 million new cases of curable STIs occur annually. HIV-1 infection rates have reached pandemic levels with an estimated 34.2 million people living with HIV-1 in 2011 and 2.5 million people becoming newly infected with HIV this year. ${ }^{1}$ The advent of resistant mutations to antiretroviral drug compounds and classes may endanger the success of HIV-1 treatment and accelerate disease progress worldwide. ${ }^{2-4}$ It is known that more than $80 \%$ of adult HIV-1 infections have resulted from heterosexual intercourse. Females are particularly susceptible to heterosexual transmission of HIV-1 due to substantial mucosal exposure to seminal fluids. While condoms are 
effective in preventing HIV-1 infection, they provide a lesser degree of protection against genital human papillomavirus (HPV), herpes simplex virus (HSV), and bacterial and fungal infections since they may be transmitted across surfaces not covered or protected by the condom. Moreover, a small percentage of people have allergic reactions to latex, plus a relatively high rate of condom breakage or slippage is observed. Two to three percent of women will test positive for semen residue after intercourse with a condom. ${ }^{5}$ Thus, there is a high demand to develop new technologies that are not only able to mechanistically separate pathogens from the host, but are also able to directly disrupt the infectivity of the pathogens and effectively control the sexual transmission of infections including HIV-1 and genital herpes.

Recently, numerous studies have shown that nanotechnology is an emerging multidisciplinary field, which will greatly help in overcoming the limitations of treatment. Particularly, inorganic materials like gold, silver, iron, titanium, copper, silica, and zinc oxide have been used to fabricate nanoparticles (NPs) for several pharmaceutical applications, including cancer therapeutics, ${ }^{6,7}$ cellular and biomolecular labels, ${ }^{8}$ and biosensors. ${ }^{9}$ Among these, silver NPs (Ag-NPs) have received considerable attention as antimicrobial agents due to their strong antibacterial, ${ }^{10}$ antifungal, ${ }^{11}$ and antiviral activities. ${ }^{12,13}$ Antiviral activity of Ag-NPs has been demonstrated against several types of viruses, including hepatitis $\mathrm{B}, \mathrm{HSV}$, respiratory syncytial virus, and monkey pox virus. ${ }^{12-14}$ Moreover, several studies have demonstrated that nanosilver has antiviral activity against HIV-1 in vitro. ${ }^{13-15}$ Silver NPs were shown to be effective against a wide range of HIV-1 strains in vitro, including laboratory strains, clinical isolates, $M$ and $\mathrm{T}$ tropic strains, and antiretroviral drug-resistant strains. ${ }^{15}$ By binding to HIV-1 gp120, Ag-NPs prevent CD4-mediated viral membrane fusion and thus inhibit the virus's entry and infectivity. ${ }^{14}$ In addition, Ag-NPs were also found to inhibit postentry stages of HIV-1, ${ }^{15}$ indicating that Ag-NPs may act at multiple stages of the HIV-1 life cycle.

In this study, our research group developed an Ag-NPscoated PUC and investigated its antimicrobial activity toward HIV-1 and HSV. Our results showed that after HIV-1 contacted an Ag-NPs-coated PUC, the infectiousness of the virus could be completely inactivated. Both M-tropic and T-tropic viruses were highly susceptible to Ag-NPscoated PUCs. Similarly, Ag-NPs-coated PUC exhibited strong inactivating activity against both HSV-1 and HSV-2. In addition, we have also shown that the Ag-NPs-coated PUC was able to inhibit the growth of bacteria and fungi.
Overall, this study provides evidence that, in addition to its ability to mechanically isolate microbes from host cells, the Ag-NPs-coated PUC is also able to inactivate the infectiousness of microbes, providing another defense line against sexually transmitted diseases.

\section{Materials and methods Synthesis of Ag-NPs and fabrication of Ag-NPs-coated PUC}

The synthesis of Ag-NPs was performed following the Turkevich method. ${ }^{16}$ Briefly, a $100-\mathrm{mL}$ sample of aqueous trisodium citrate $(7.0 \mathrm{mM})$ was prepared in a glass flask, first brought to a boil while being stirred, and then $1.0 \mathrm{~mL}$ of $0.1 \mathrm{M}$ aqueous silver nitrate was added to start the reaction. The color of the reaction solution was observed to change generally from colorless to yellow, then turbid. No further changes indicated the reaction was completed. Then, the PUCs were soaked in Ag-NP solution overnight. Partial coverage can be achieved for shorter exposure times. Each Ag-NPs-coated PUC was washed extensively with water to remove any adsorbed ions such as citrate and air-dried.

The Ag-NPs-coated PUC was characterized by electron microscopy, diffused reflectance spectroscopy, and Fourier transform-infrared spectroscopy (FTIR). Scanning electron micrographs (SEMs) were taken with an FEI Quanta FEG 200 high-resolution SEM (HrSEM; FEI, Hillsboro, OR) with an energy-dispersive analysis of X-ray (EDAX) attachment. Ultraviolet-visible-near-infrared diffuse reflectance spectra were recorded with a Varian 5E spectrometer (Varian Medical Systems, Palo Alto, CA). FTIR studies were conducted using a Spectrum One spectrometer (PerkinElmer, Waltham, MA).

\section{Cell toxicity of Ag-NPs-coated PUC}

The 4-[3-(4-iodophenyl)-2-(4-nitrophenyl)-2H-5-tetrazolio]1,3-benzene disulfonate (WST-1) assay (Roche, Mannheim, Germany) was used to measure the growth kinetics of HeLa cells after exposure to the Ag-NPs-coated PUC. Briefly, $1 \times 10^{6}$ HeLa cells in 100- $\mu \mathrm{L}$ culture medium were exposed to an AgNPs-coated PUC piece $\left(1 \mathrm{~cm}^{2}\right)$ or noncoated PUC $\left(1 \mathrm{~cm}^{2}\right)$ in a 24-well plate for 10, 30, and 180 minutes. Then, the PUC piece was removed, and cells and medium were cultured in a 96-well plate at a density of $1 \times 10^{3}$ cells/well and incubated at $37^{\circ} \mathrm{C}$, $5 \% \mathrm{CO}_{2}$. At different time intervals, the WST-1 $(10 \mu \mathrm{L} /$ well $)$ was added in the culture and incubated for 4 hours at $37^{\circ} \mathrm{C}$. After shaking thoroughly for 1 minute, the absorbance was measured at $490 \mathrm{~nm}$ using a POLARstar OPTIMA microplate reader (BMG Labtech, Offenberg, Germany). 
To test the toxic effect of Ag-NPs-coated PUCs in human 293 T cells and the C8166 T lymphoid cell line, $0.5 \times 10^{6}$ cells of either 293 T or C8166 T cells were incubated with an AgNPs-coated PUC or noncoated PUC for 180 minutes. Then, the PUC piece was removed, and cells and medium were cultured in a 96 -well plate at a density of $4 \times 10^{3}$ cells/well and incubated at $37^{\circ} \mathrm{C}, 5 \% \mathrm{CO}_{2}$. The growth kinetics of cells were monitored using the WST-1 assay for up to 4 days.

\section{Cell culture and viruses}

Human embryonic kidney 293 T cells, human cervical cancer cells (HeLa cell), HeLa-CD4-CCR5-LTR- $\beta$-gal cells, and African green monkey kidney cells-Vero E6 cells were maintained in Dulbecco's modified Eagle's medium (DMEM) supplemented with 10\% fetal bovine serum (FBS), 100 units/mL penicillin, and $100 \mu \mathrm{g} / \mathrm{mL}$ streptomycin. Human C8166 T lymphoid cells were maintained in RPMI1640 medium containing $10 \% \mathrm{FBS}$ and $100 \mathrm{U} / \mathrm{mL}$ penicillin and $100 \mu \mathrm{g} / \mathrm{mL}$ streptomycin. HIV-1 pNL4.3-GFP + virus stock was produced by transfecting 293 T cells with pNL4.3GFP proviral DNA, as described previously. ${ }^{17}$ Virus titer was quantified using HIV-1 p24 Antigen Capture Assay Kit (purchased from the NCI-Frederick AIDS Vaccine Program; NCI-Frederick Cancer Research and Development Center, Frederick, MD). HSV-1 and HSV-2 ${ }^{18}$ were generously provided by Dr Alberto Severini from the Canadian Science Centre for Human and Animal Health located in Winnipeg, Canada. These viruses were propagated in Vero E6 cells and the viral titer, expressed as plaque-forming units (PFU) per well, was determined by plaque assay. ${ }^{19}$

\section{Anti-HIV infection assays}

To test the effect of the Ag-NPs-coated PUC on HIV-1 infection, $200 \mu \mathrm{L}$ of RPMI containing pNL4.3-GFP virus (5 ng of GagP24) was incubated with a piece $\left(1 \mathrm{~cm}^{2}\right)$ of the Ag-NPs-coated PUC or noncoated PUC $\left(1 \mathrm{~cm}^{2}\right)$ for $10,30,60$, and 120 minutes with continuous shaking. Meanwhile, the virus-containing medium without incubation with any PUC was used as a positive control. After incubation, the virus-containing mediums were collected and used to infect $0.2 \times 10^{6} \mathrm{CD} 4+\mathrm{C} 8166 \mathrm{~T}$ cells. HIV-1 infectivity was assayed by the observation of levels of viruses-induced syncytium formation and HIV-1-infected (GFP+) cells under both optical and fluorescence microscopy after 3 days of infection. At different time intervals after infection, HIV-1 replication levels were also monitored by measuring the HIV-1 Gag antigen levels present in the supernatants using an HIV-1 p24 gag ELISA.
To determine if the inactivation of $\mathrm{HIV}-1$ required the direct interaction between virus and the Ag-NPs-coated PUC, $200 \mu \mathrm{L}$ of RPMI was incubated with the Ag-NPs-coated PUC $\left(1 \mathrm{~cm}^{2}\right)$ or noncoated PUC $\left(1 \mathrm{~cm}^{2}\right)$ in a 24-well plate with continuous shaking. After 30 minutes, $200 \mu \mathrm{L}$ of RPMI (A) was transferred to an empty well (A), and $200 \mu \mathrm{L}$ of fresh RPMI (B) was added to the original well (B) containing the NPs-coated or noncoated PUC. Then, pNL4.3-GFP virus (5 ng of GagP24) was added to the wells A and B and incubated at $37^{\circ} \mathrm{C}$ for 30 minutes with continuous shaking. After incubation, the virus-containing mediums were collected and used to infect $0.2 \times 10^{6} \mathrm{CD} 4+\mathrm{C} 8166 \mathrm{~T}$ cells. After 3 days of infection, HIV-1 infection was examined by checking for HIV-induced syncytium formation and HIV-1 infected (GFP+) cells and by the measurement of the HIV-1 Gag antigen levels present in the supernatants.

Meanwhile, the effect of the Ag-NPs-coated PUC on M-tropic HIV-1 virus was tested by incubating $200 \mu \mathrm{L}$ medium containing macrophage $(\mathrm{M})$-tropic virus $\mathrm{pNL} 4.3-\mathrm{Bal}$ (50 ng of GagP24) with the Ag-NPs-coated PUC (1 $\left.\mathrm{cm}^{2}\right)$, noncoated PUC $\left(1 \mathrm{~cm}^{2}\right)$ for 10, 30, and 60 minutes with continuous shaking. After incubation, $50 \mu 1$ of the virus-containing medium was added into each well of a 96-well plate containing HeLa-CD4-CCR5- $\beta$-Gal cells. After 48 hours postinfection, the numbers of infected cells ( $\beta$-Gal positive cells) in each well were evaluated by the multinuclear activation of a galactosidase indicator (MAGI) assays. ${ }^{21,22}$

\section{Anti-HSV infection assay}

To determine the anti-HSV activity of Ag-NPs-coated PUCs, confluent Vero-E6 cell monolayers were prepared with $5 \times 10^{4}$ cells/well in 24-well plates until they reached at least $95 \%$ confluence. Meanwhile, $200 \mu \mathrm{L}$ of DMEM containing different amounts of HSV-1 or HSV-2 (50-500 pfu) were first incubated with the Ag-NPs-coated PUC $\left(1 \mathrm{~cm}^{2}\right)$ or noncoated PUC $\left(1 \mathrm{~cm}^{2}\right)$ for 30 minutes with shaking every 5 minutes. Then, the virus-containing mediums were collected and used to infect Vero-E6 cells at $37^{\circ} \mathrm{C}$ in $5 \% \mathrm{CO}_{2}$ condition. After 48 hours, the viral infectivity was analyzed by observation of the virus-induced cytopathic effects (lesions) under microscope.

\section{Source of microorganism}

Pure cultures of Escherichia coli (American Type Culture Collection [ATCC] 8739), Staphylococcus aureus (ATCC 6538), Microccocus luteus (ATCC 4698), Klebsiella pneumoniae (ATCC 13883), Candida tropicalis (ATCC 13803), C. krusei (ATCC 14243), C. glabrata (ATCC 
15126), and C. albicans (ATCC 10231) were obtained from ATCC (Manassas, VA).

\section{Assessment of antimicrobial activity of Ag- NPs-coated PUC against E. coli, S. aureus, M. luteus, K. pneumonia, and Candida spp.}

The Ag-NPs-coated and noncoated PUCs were disinfected by ultraviolet $\mathrm{C}$ irradiation for 1 minute. Then, Ag-NPs coated and noncoated material $\left(2 \mathrm{~cm}^{2}\right)$ were inoculated in $3 \mathrm{~mL}$ Müller-Hilton broth with fresh E. coli, S. aureus, M. luteus, K. pneumoniae, and Candida spp. (C. tropicalis, C. krusei, C. glabrata, and C. albicans) at a concentration of $10^{5}$ colonyforming units per $\mathrm{mL}$ ( $\mathrm{cfu} / \mathrm{mL}$ ). After 10 minutes incubation in shaking condition, Ag-NPs-coated or noncoated PUC materials were removed from the test tubes and the sample without condom material was used as a positive control. All test tubes were incubated at $37^{\circ} \mathrm{C}$ for 24 hours. After incubation, $100 \mu \mathrm{L}$ of the sample was drawn from each test tube and inhibition of growth was determined by measuring optical density at $600 \mathrm{~nm}$ using a POLARstar OPTIMA microplate reader.

\section{Results}

\section{Production and characterization of Ag-NPs-coated PUC}

First, we have developed an Ag-NPs-coated PUC by soaking a PUC in aqueous Ag-NPs solution, as described in Materials and Methods. The saturated binding of NPs on polyurethane gives a golden yellow color, as shown in Figure 1A. The Ag-NPs binding at the surface of the condom was analyzed by high-resolution scanning electron microscopy (HrSEM). HrSEM micrographs of the PUC and the PUC coated with Ag-NPs showed the incorporation of Ag-NPs on the surface of the condom, as shown in Figure $1 \mathrm{C}$ and D. The PUC maintained its morphology upon coating with Ag-NPs (Figure 1B). It is observed from the micrograph that most of the Ag-NPs are in the range of 30-60 nm in size. Elemental composition of the NPs-coated PUC was analyzed by energydispersive X-ray spectroscopy. Figure 1D showed the optical absorption peak is approximately at $3 \mathrm{keV}$.

\section{Spectroscopy analysis of Ag-NPs-coated PUC}

Diffused reflectance spectrum of polyurethane coated with Ag-NPs shows a peak at $430 \mathrm{~nm}$ (Figure 2A). FTIR Spectra of the coated and noncoated samples in the region of relevance are shown in Figure 2B. There is a slight shift in the $\mathrm{N}$ peak upon NP coating, while all the other peaks remain unchanged. The binding is due to the interaction between the nitrogen of the $\mathrm{NH}$ bond of the polyurethane and Ag-
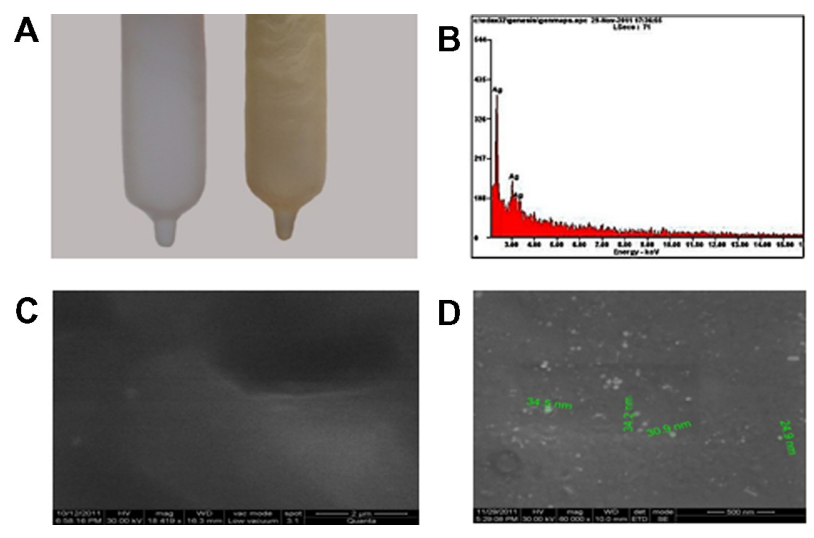

Figure I Photographs of a PUC (A, left) and an Ag-NPs-coated PUC (A, right). Yellow color in (B) is due to the saturation coverage of Ag-NPs. Spot EDAX analysis (B) confirmed the presence of elemental silver on the surface of the PUC. SEM of PUC (C) and PU coated with Ag-NPs (D).

Abbreviations: Ag-NPs, silver nanoparticles; EDAX, energy-dispersive analysis of X-ray; PUC, polyurethane condom; SEM, scanning electron microscopy.

NPs. ${ }^{16}$ It appears that not all the polyurethane is involved in bonding, as part of the -NH stretch is unaffected. Further, the coating method does not affect the chemical properties of the material. The coated condom was washed in distilled water six times (keeping condom in water for 20 minutes per wash) and the coated condom was air-dried.

\section{Toxicity of Ag-NPs-coated PUC}

The same amounts of human cervical cancer cells (HeLa cells) were exposed to the Ag-NPs-coated PUC or noncoated PUC for different times (10, 30, and 180 minutes). Then, the Ag-NPs-coated PUC or noncoated PUC was removed and the growth of HeLa cells was monitored at different days postexposure by using a cell proliferation (WST-1) assay. The results showed that, after being exposed to Ag-NPs-coated PUC for 3 hours, the growth of HeLa cells was not affected at up to 96 hours of culture

A

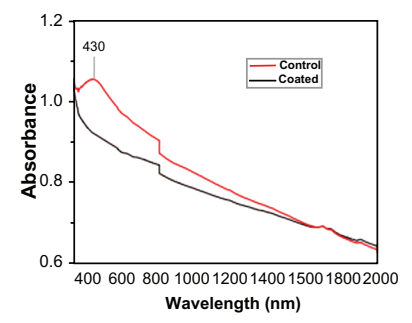

B

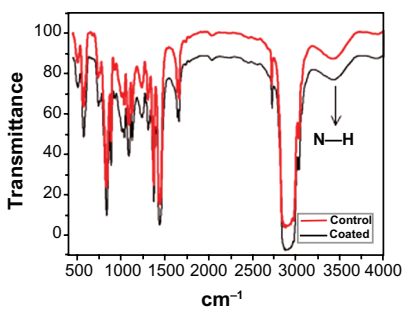

Figure 2 (A) Diffused reflectance spectra of PUC before (control) and after coating with Ag-NPs. (B) FTIR spectra of noncoated (control) and coated PUC.

Note: The slight shift in $\mathrm{N}-\mathrm{H}$ peak after coating with Ag-NPs clearly indicates binding is due to the interaction between the nitrogen of the $N(H)$ bond of polyurethane and Ag-NPs.

Abbreviations: Ag-NPs, silver nanoparticles; FTIR, Fourier transform infrared; PUC, polyurethane condom. 

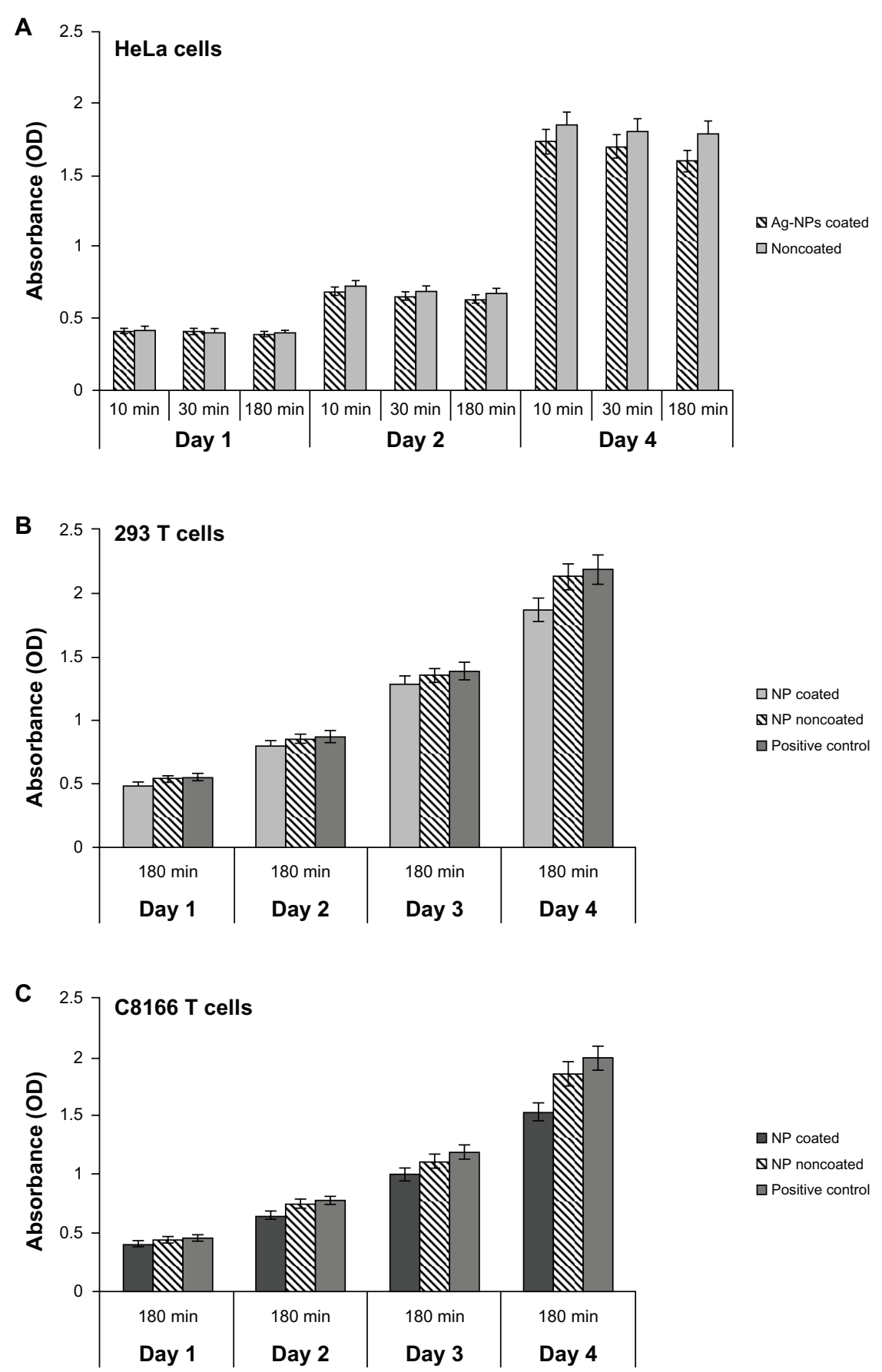

$\square$ NP coated

$\triangle \mathrm{NP}$ noncoated

$\square$ Positive contro

Figure 3 Toxicity of Ag-NPs-coated PUC. HeLa cells (A) were incubated with Ag-NPs-coated or noncoated condom for 10, 30, and 180 minutes. A WST assay was performed to determine the growth of cell populations at day I, day 2, and day 4 after incubation with Ag-NPs-coated or noncoated condom. To test the toxic effect of the Ag-NPs-coated PUC in human $293 \mathrm{~T}$ cells and C8166 T line, $0.5 \times 10^{6}$ cells of $293 \mathrm{~T}$ (B) or C8I66 T cells (C) were incubated with an Ag-NPs-coated PUC or noncoated PUC for 180 minutes. Then, the PUC was removed and the cell growth was monitored using the cell proliferation (WST-I) assay.

Abbreviations: Ag-NPs, silver nanoparticles; PUC, polyurethane condom; WST, 4-[3-(4-iodophenyl)-2-(4-nitrophenyl)-2H-5-tetrazolio]-I,3-benzene disulfonate.

periods (Figure 3A). In addition, the toxic effect of the Ag-NPs-coated PUC was further tested in human $293 \mathrm{~T}$ cells and in the C8166 T lymphoid cell line. Briefly, each cell line $\left(0.5 \times 10^{6}\right.$ cells $)$ was incubated with the Ag-NPscoated PUC or noncoated PUC for 180 minutes. Then, the PUC was removed and cells continued to grow in medium.
At different days, cell growth was monitored by using the WST-1 assay. The results showed that, after exposure to the Ag-NPs-coated PUC for 3 hours, the growth of 293 T cells and $\mathrm{C} 8166 \mathrm{~T}$ cells was not affected at up to 4 days of culture periods (Figure 3B and C). All of these results suggest that at up to 3 hours of contact, the Ag-NPs-coated PUC does 
not have significant effect on the viability and growth of HeLa, $293 \mathrm{~T}$ and, C8166 T cells.

\section{Exposure of HIV-I to Ag-NPs-coated PUC} significantly diminished both T-tropic and M-tropic virus infectivity

We next tested whether the Ag-NPs-coated PUC was able to inhibit HIV-1 infectivity through the contact of HIV-1 to the Ag-NPs-coated PUC or noncoated PUC. Results showed that the exposure of pNL4.3-GFP virus to the noncoated PUC did not significantly affect its infectivity as compared to the positive control (Figure 4A). However, when pNL4.3-GFP virus contacted the Ag-NPs-coated PUC, the virus infectivity was drastically inhibited (Figure 4A). These results clearly revealed that even for a 10-minute period of exposure time, the majority of pNL4.3-GFP virus lost the ability to infect CD4 + T cells (Figure 4A, left upper panel). The inhibitory effect of the Ag-NPs-coated PUC on HIV-1 infectivity was further analyzed by optical microscopy for HIV-induced syncytium formation (Figure 4B, a-d, i-1) and HIV-infected $(\mathrm{GFP}+$ ) cells (Figure 4B, e-h, m-p). When pNL4.3-GFP virus contacted the Ag-NPs-coated PUC for 10 minutes, only a few

A
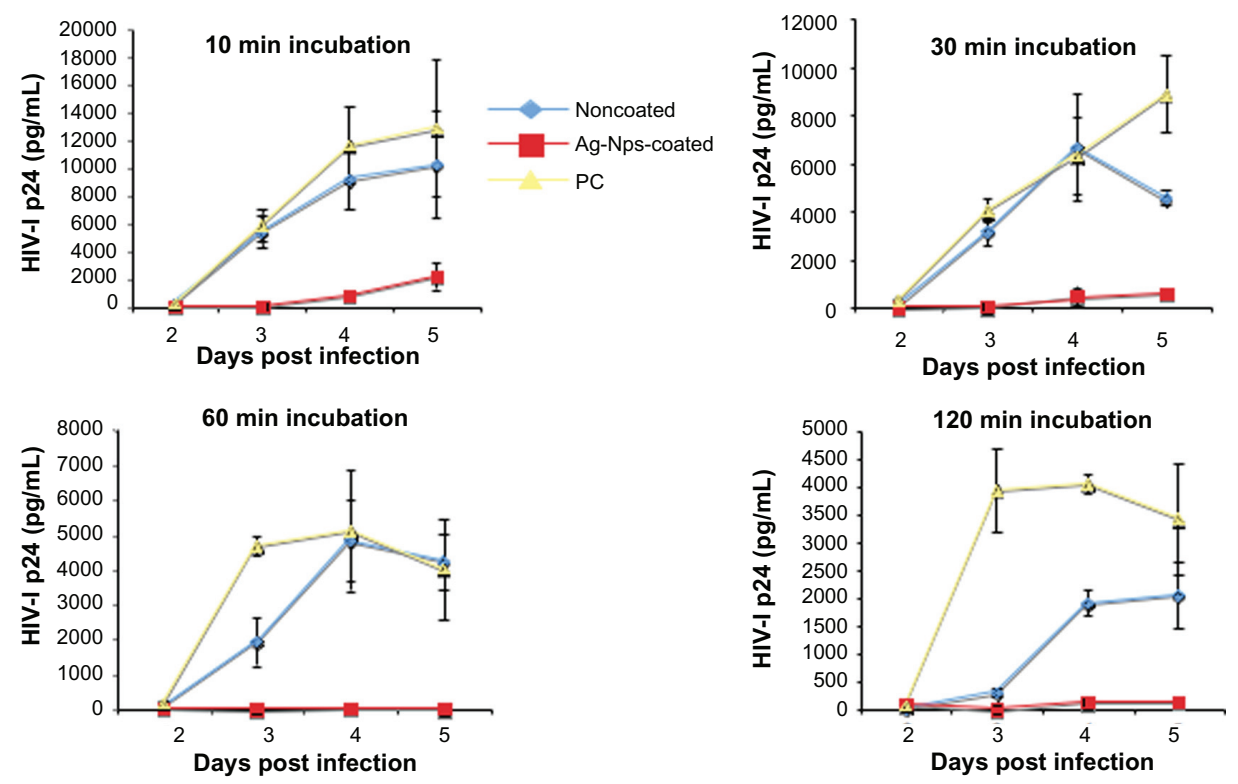

B

\begin{abstract}
Incubation time $10 \mathrm{~min}$
\end{abstract}

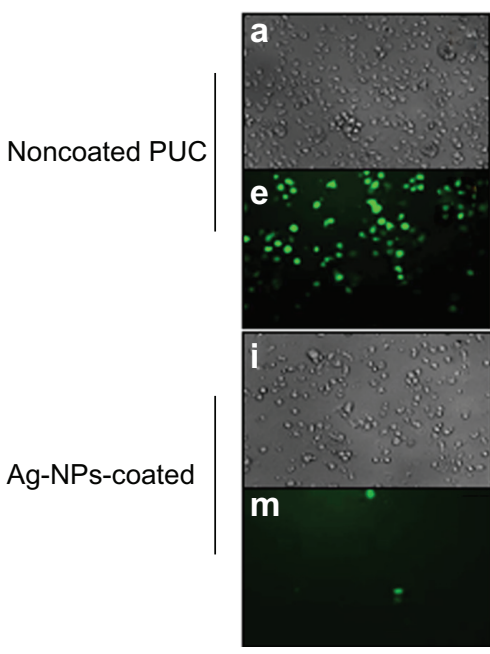

$30 \mathrm{~min}$

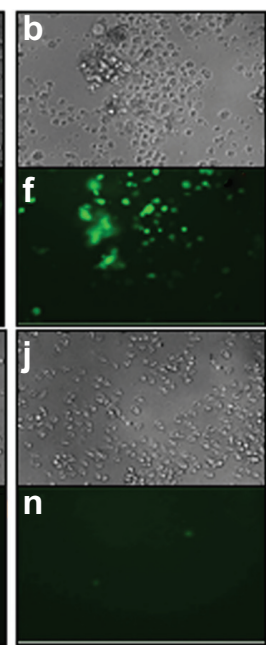

$60 \mathrm{~min}$

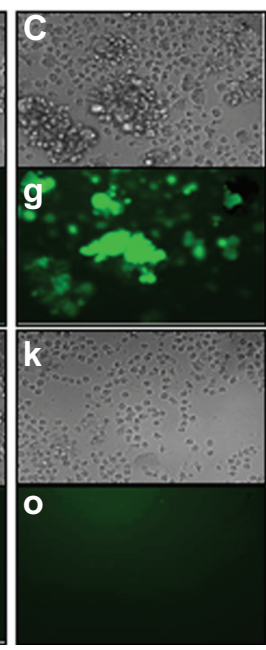

$120 \mathrm{~min}$

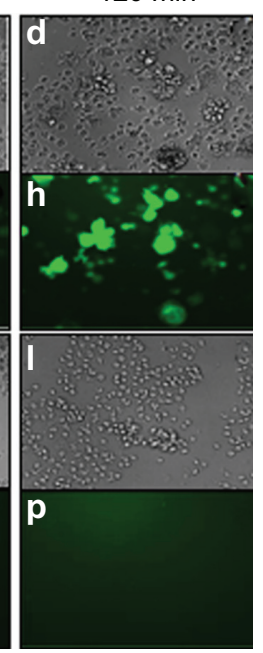

Figure 4 The inhibitory effect of the Ag-NPs-coated PUC on T-tropic HIV-I infection in C8I66 T cells. pNL4.3 GFP + virus was first incubated with the Ag-NPs-coated or noncoated condom for 10, 30, 60, or 120 minutes, in RPMI with consistent shaking. Then, the RPMI-containing viruses were used to infect CD4 + C8I66 cells. The same amount of pNL4.3-GFP + virus without incubation with condom was used as positive control. At different days postinfection, HIV-I-infected GFP positive cells were observed under fluorescence microscopy (B) and the levels of HIV-I Gag P24 in supernatants were measured by ELISA assay (A).

Abbreviations: Ag-NPs, silver nanoparticles; GFP, green fluorescent protein; HIV, human immunodeficiency virus; PUC, polyurethane condom. 
GFP + cells were detected, but no syncytium formation was observed (Figure 4B, i and m). As expected, the inhibitory effect increased after 30 minutes contact with the Ag-NPscoated PUC and there was no GFP + cell or syncytium formation observed (Figure 4B, $\mathrm{j}$ and $\mathrm{n}$ ). In contrast, after being exposed to the noncoated PUC for 10 or 30 minutes, HIV-1 maintained its infection ability and a massive viral infection in C8166 T cells was observed (Figure 4B, a, b, and e, f). Next, we further tested whether macrophage (M)-tropic virus is also susceptible to the Ag-NPs-coated PUC. To do so, the culture medium containing the M-tropic pNL4.3-Bal virus was exposed to the Ag-NPs-coated PUC or noncoated PUC for 10, 30, or 60 minutes, as described before, and then the virus-containing medium was collected and incubated with HeLa- $\beta$-gal-CD4 + -CCR5 + cells. In parallel, the same amount of pNL4.3-Bal virus without incubation with the PUC was used as positive control. After 48 hours of infection, numbers of infected cells ( $\beta$-gal positive cells) in each well were evaluated by the MAGI assay. As expected, pNL4.3-Bal virus exposed to the noncoated PUC did not significantly affect their infectivity (Figure 5, compare bars 4-6 to bars 7-9). However, exposure to the Ag-NPs-coated PUC significantly inhibited the infectivity of M-tropic pNL4.3-Bal virus and at over 30 minutes of exposure, the pNL4.3-Bal virus completely lost their infection ability (Figure 5, compare bars 1-3 to bars 4-6).

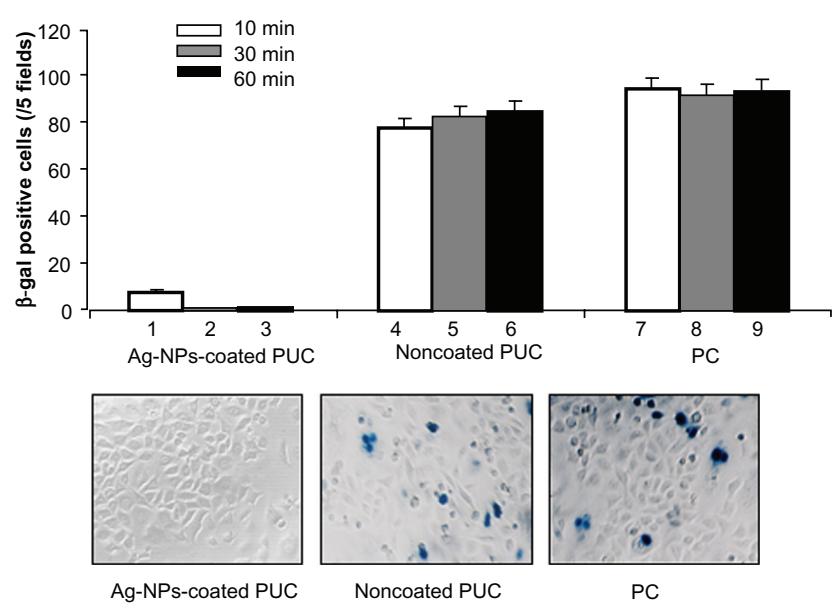

Figure 5 The inhibitory effect of Ag-NPs-coated PUC on M-tropic HIV-I infection in HeLa $\beta$-gal-CD4 + -CCR + cells.

Notes: pNL4.3-Bal virus were first incubated with Ag-NPs-coated or noncoated condom for 10, 30, or 60 minutes, in DMEM with consistent shaking. Then, the DMEMcontaining viruses were used to infect HeLa $\beta$-gal-CD4 + -CCR + cells. The same amount of $\mathrm{pNL4.3-Bal} \mathrm{virus} \mathrm{lacking} \mathrm{incubation} \mathrm{with} \mathrm{condom} \mathrm{was} \mathrm{used} \mathrm{as} \mathrm{positive} \mathrm{control.} \mathrm{After}$ 48 hours of infection, MAGI assay was used to detect HIV-I infected cells.

Abbreviations: Ag-NPs, silver nanoparticles; DMEM, Dulbecco's modified Eagle's medium; M, macrophage; MAGI, multinuclear activation of a galactosidase indicator; HIV, human immunodeficiency virus; PUC, polyurethane condom.

\section{Direct exposure of HIV-I to Ag-NPs-coated PUC} is required to inactivate the virus infectivity

The above results show that HIV-1 infectivity was diminished when viruses were exposed to the Ag-NPs-coated PUC. However, we still do not know whether this anti-HIV activity is due to the action of bound Ag-NPs or the NPs released from the PUC. To address this question, we incubated the Ag-NPscoated PUC in a 24-well plate containing $200 \mu \mathrm{L}$ RPMI1640 medium with continuous shaking. After 30 minutes, the medium was transferred from the well containing the Ag-NPs-coated PUC to an empty well, and the same amount of fresh medium was added to the Ag-NPs-coated PUC-containing well. Then, the equal amount of pNL4.3 GFP + virus (5 ng of GagP24) was added to each well and incubated for 30 minutes. In parallel, the noncoated PUC was used as a control. After incubation, viruses from each well were collected and used to infect CD4 + C8166 T cells and their infection levels were monitored by measuring the level of Gag-p24 in the supernatant after 48 hours of infection (Figure 6A).

As expected, viruses lost their infection ability after direct exposure to the Ag-NPs-coated PUC (Figure 6A, bar 1; B, a and $b$ ). However, the virus exposed to the medium that was pre-incubated with the Ag-NPs-coated PUC for 30 minutes still reflected a high level of infection, although it was about $40 \%$ lower than the virus from the noncoated PUC samples (Figure 6A, compare bars 2 to 4; B, d to h). Such reduced HIV infection could be due to the fact that a very small amount of Ag-NPs could be released from the PUC under the continuously shaking condition, which may interfere with the HIV-1 infectivity.

\section{Inhibition of HSV-I and HSV-2 infection by Ag-NPs-coated PUC}

We next tested the antiviral effect of the Ag-NPs-coated PUC against HSV1/2 infections. A sample of $200 \mu \mathrm{L}$ of the DMEM containing HSV-1 or HIV-2 viruses (50-500 $\mathrm{pfu}$ ) was incubated with a $1-\mathrm{cm}^{2}$ piece of the Ag-NPscoated PUC or the noncoated PUC for 30 minutes. Then, the medium was collected and used to infect Vero-E6 cells, and at 48 hours postinfection, the virus-induced cytopathic effects were recorded, as indicated in Figure 7. Results showed that, after exposure to an noncoated PUC, both HSV-1 and HSV-2 maintained their infectivity as efficiently as nontreated viruses (Figure 7A and B, compare c and $\mathrm{d}$ to $\mathrm{e}$ and $\mathrm{f}$ ). In contrast, when viruses contacted with an Ag-NPs-coated PUC, the infectivity of both HSV-1 and HSV-2 was completely lost and there was no sign 


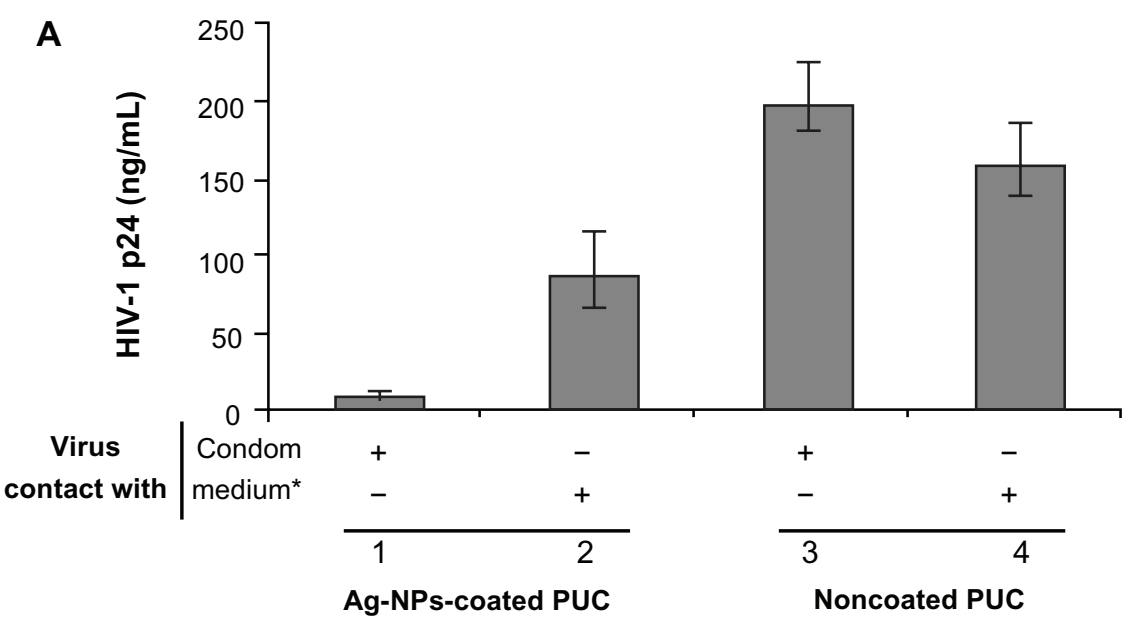

B

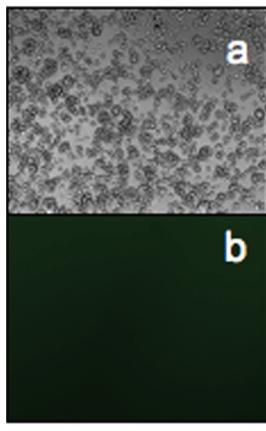

Ag-NPs PUC

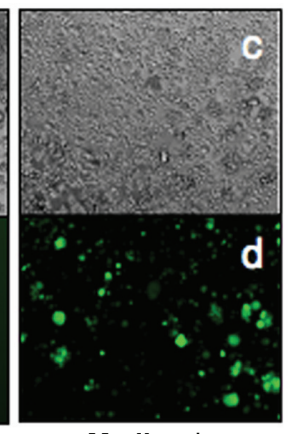

Medium*

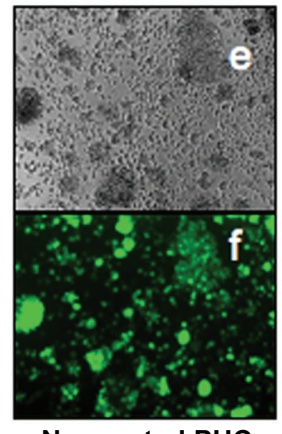

Noncoated PUC

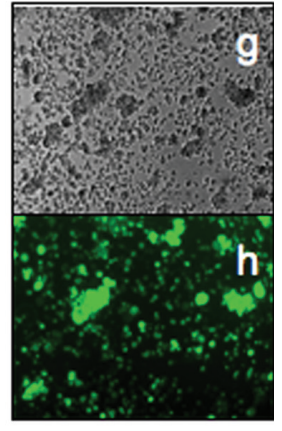

Medium*

Figure $6 \mathrm{Ag}$-NPs that tightly bind to a polyurethane condom play the major role in inactivating HIV-I infectivity. PNL4.3 GFP + virus (5 ng of GagP24) was added to wells in a 24-well plate that contains RPMI (medium) after being incubated with an Ag-NPs-coated or noncoated PUC for 30 minutes, or wells containing both RPMI and a piece of Ag-NPs-coated or noncoated PUC $\left(\mathrm{I} \mathrm{cm}^{2}\right)$. After incubating for 30 minutes with continuous shaking, the virus-containing mediums were collected and used to infect $0.2 \times 10^{6}$ CD4 + C8I66 T cells. At day 3 postinfection, HIV-I infectivity was examined by the measurement of the HIV-I Gag antigen levels present in the supernatants (A) and by the observation of the virus-mediated syncytium formation and HIV-I-infected GFP + cells (B).

Note: *RPMI medium was incubated with Ag-NPs-coated or noncoated PUC for 30 minutes, and then the PUC was removed.

Abbreviations: Ag-Nps, silver nanoparticles; HIV, human immunodeficiency virus; GFP, green fluorescent protein; RPMI medium, Roswell Park Memorial Institute medium; PUC, polyurethane condom; $\mathrm{cm}$, centimeters.

of infection in Vero-E6 cells after 2 days of incubation (Figure 7B, a and c).

\section{Anti-bacterial and anti-fungal activities mediated} by Ag-NPs-coated PUC

In addition to HIV-1 and HSVs, we further tested the antibacterial and anti-fungal activities of the Ag-NPs-coated PUC. First, fresh E. coli, S. aureus, M. luteus, K. pneumoniae, Candida tropicalis, C. krusei, C. glabrata, and C. albicans were grown in separate Muller Hilton broths. To test the inhibitory effect of the Ag-NPs-coated PUC on the growth of each bacterial and fungal agent, the Ag-NPs-coated and noncoated PUCs $\left(2 \mathrm{~cm}^{2}\right)$ were inoculated in 3-mL Muller Hilton broth with a concentration of $10^{5} \mathrm{cfu} / \mathrm{mL}$. After 10 minutes incubation in shaking condition, the Ag-NPs-coated or noncoated PUCs were removed and all the test tubes continued the culture at $37^{\circ} \mathrm{C}$ for $24 \mathrm{~h}$. Then, $100 \mu \mathrm{L}$ of the sample was collected from each test tube and the inhibition of the
Ag-NPs-coated PUC on bacterial growth was determined by using the POLARstar OPTIMA microplate reader. The analyses showed significant inhibitory activity of the Ag-NPscoated PUC against all tested microorganisms, as shown in Figure $8 \mathrm{~A}$ and $\mathrm{B}$, while the noncoated PUC did not show any antimicrobial activity.

\section{Discussion}

Sexually transmitted infection (STI) prevention is an important reason for condom use. However, a condom does not offer complete protection against HIV-1, HSV-2, human papilloma virus, or bacterial and fungal sexual infections in both men and women. ${ }^{24}$ The nonoxynol-9-coated condom was once believed to offer additional protection against STIs. However, recent studies have shown that the use of nonoxynol-9-coated condoms induces inflammation and ulceration to genital mucosa, thereby increasing the probability of transmitted infectious agents. ${ }^{25,26}$ Till now 
Inhibition of Ag-NPs-coated PUC on HSV induced cytopathic effect

\begin{tabular}{lccccc}
\hline & \multicolumn{3}{c}{ HSV-1 } & \multicolumn{2}{c}{ HSV-2 } \\
Virus titer (pfu) & 50 & 500 & 50 & 500 \\
\hline Ag-NPs-coated PUC & - & - & - & - \\
Noncoated PUC & $>+++$ & $>++++$ & $>+++$ & $>++++$ \\
\hline
\end{tabular}
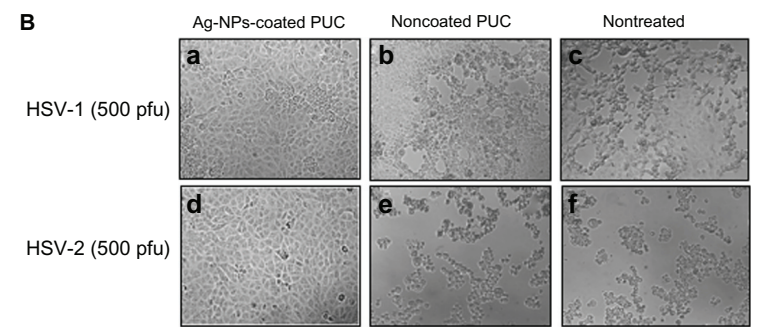

Figure 7 The inhibitory effect of the Ag-NPs-coated PUC on HSV infection.

Notes: Different titers (pfu) of HSV-I or HSV-2 virus in $200 \mu \mathrm{L}$ of DMEM were first incubated with the Ag-NPs-coated PUC or noncoated condom for 30 minutes. Then, the virus-containing medium was used to infect Vero-E6 cells in 24-well plates. After 48 hours, the virus-induced cytopathic effects (lesion) were observed under microscope and the cytopathic lesions in five views were counted and calculated as lesions per well. (-) no HSV-induced cytopathic lesion; (+) average two cytopathic lesions/view; (++) two cytopathic lesions/view; (+++) three cytopathic lesions/view; $(++++)$ four cytopathic lesions/view.

Abbreviations: Ag-NPs, silver nanoparticles; UC, polyurethane condom; HSV, herpes simplex virus; DMEM, Dulbecco's modified Eagle's medium; PFU, plaque-forming units.

there has been no single condom with additional protection against sexually transmitted infections available on the market. Previous studies have shown that Ag-NPs exhibit anti-inflammatory effect, ${ }^{27,28}$ a broad spectrum of antibacterial and antiviral activities, and are relatively nontoxic to the human body at low concentration. ${ }^{12,29-31}$ Thus, in this study, we have developed an Ag-NPs-coated condom product and further tested its inhibitory activities against HIV-1, HSV-1/2, and other bacterial and fungal pathogens.

The spectroscopic analyses of the silver Ag-NPs-coated PUC showed a peak at $430 \mathrm{~nm}$, which is due to excitation of the surface plasmon resonance of Ag-NPs (Figure 2A) and optical absorption peak in the EDAX at $3 \mathrm{keV}$ clearly confirmed the presence of nanocrystalline elemental silver. ${ }^{22}$ The FTIR analysis shows most of the functional group of polyurethane remained intact after coating with Ag-NPs (Figure 2B). Thus, this method preserves the original qualities of the manufactured condom. Importantly, coating takes place on both the inner and outer surfaces of the condom, thus providing a double protection against pathogens.

Prior to testing its antimicrobial efficacy, an evaluation of whether the contact of the Ag-NPs-coated PUC on human
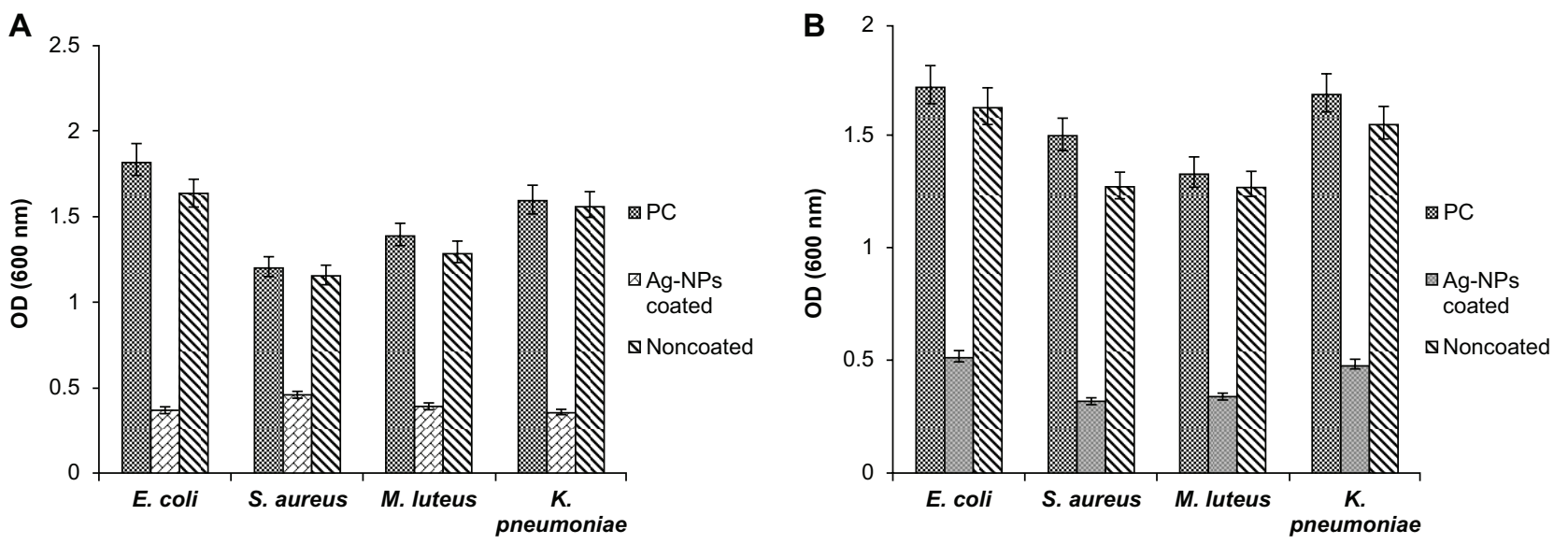

Figure 8 The inhibitory effect of the Ag-NPs-coated PUC on (A) bacteria (B) Candida spp. Ag-NPs-coated PUC $\left(2 \mathrm{~cm}^{2}\right)$ or noncoated PUC (2 cm $\left.{ }^{2}\right)$ material was inoculated in 3-mL Muller Hilton broth containing fresh E. coli, S. aureus, M. luteus, K. pneumoniae, or Candida spp. at a concentration of $10^{5}$ colony-forming units per $\mathrm{mL}$ (cfu/mL). Notes: After 10 minutes incubation in shaking condition, Ag-NPs-coated or noncoated PUC samples were removed from the test tubes and the bacteria cultures were incubated at $37^{\circ} \mathrm{C}$ for 24 hours. The sample without condom material inoculated was treated as positive control (PC). The growth inhibition of bacteria was determined by measuring optical density at $600 \mathrm{~nm}$ using the ELISA plate reader.

Abbreviations: Ag-NPs, silver nanoparticles; PUC, polyurethane condom; ELISA, enzyme-linked immunosorbent assay; cm, centimeter; nm, nanometer. 
cells would cause any detrimental effect was required. Our results indicate that the Ag-NPs-coated PUC did not significantly affect HeLa cells, 293 T, and C8166 T cells' survival and growth after the cells made contact with the Ag-NPscoated PUC for up to 3 hours (Figure 3). However, whether contact with the Ag-NPs-coated PUC for a longer time would result in cell damage requires further investigation. Notably, Lara et al demonstrated that a high concentration of polyvinyl pyrolidone-coated Ag-NPs $(0.6 \mathrm{mg} / \mathrm{mL})$ were only cytotoxic to a small population of cells, affecting the viability of $20 \%$ of the cells in the cervical explants. ${ }^{30}$ In addition, our data show that Ag-NPs were stably bound to the polyurethane condom and were not significantly leached from the condom due to the interaction of the Ag-NPs with the nitrogen atom of the PUC. This feature could provide additional advantage for the product used at the mucosal surface since the Ag-NPs will be removed with the PUC after use.

Previous studies have proven that Ag-NPs are a broadspectrum biocide. ${ }^{31}$ However, whether Ag-NPs chemically bound to the condom still possess a potent activity against HIV and other sexually transmitted pathogens is unclear. In this study, we have clearly shown that the contact of the Ag-NPscoated PUC with both T-tropic and macrophage-tropic HIV-1 could efficiently inactivate the virus infectivity. Such potent anti-HIV activity was mediated by Ag-NPs that are bound to the PUC, but not the PUC itself since the PUC alone did not show any inhibitory effect (Figure 4). Another possibility could be that the Ag-NPs associated with the condom could be released into the medium after extensive shaking in the medium, and these released Ag-NPs could be the molecules acting on HIV-1 virions. To verify this question, we have extensively shaken the Ag-NPs-coated PUC in the medium for 30 minutes and collected the supernatant. Infection analysis revealed that the collected supernatant only showed a modest anti-HIV effect. This effect may be due to the fact that the culture medium contains different kind of ions, which may influence the small amount of silver ions released from the condom. However, the majority of anti-HIV activity was still associated with the Ag-NPs associated with the PUC (Figure 6). All of these results indicate that Ag-NPs were tightly bound to the PUC and these bound Ag-NPs were biologically active and are able to efficiently inactivate HIV-1 infectiousness. It is known that HIV-1 strains found in humans can differ widely in their pathogenicity, virulence, and sensitivity to particular antiviral drugs. ${ }^{32}$ Thus, it is necessary in our continuing study to further test whether the Ag-NPs-coated PUC could have an effective broad spectrum activity against different clades of HIV-1 as well as various drug-resistant viruses. In addition, it is interesting to explore the possible mechanism of how PUC-bound Ag-NPs achieve such potent anti-HIV activity. One possible mechanism could be due to the direct transfer of silver ions from oxidized NPs to biological targets such as virus membrane proteins, like gp120 and gp41. Also, it should be noted that a very small amount of silver ions could also be released from the Ag-NPs-coated PUC (Figure 6) and it is possible that these very low levels of silver ions at the mucosal surface would enhance the anti-HIV efficiency during and even after the use of the Ag-NPs-coated PUC. More detailed studies will address these questions.

Herpes simplex virus (HSV) is another common infectious agent that occurs worldwide and infects humans of all ages. ${ }^{12}$ The outcome of HSV-1 infection includes a wide variety of clinical manifestations, ranging from asymptomatic infection to oral cold sores and severe encephalitis while HSV-2 causes genital herpes. Over the last decade, due to the growth of immunocompromised patients and an occurrence of HIV infection, an increase in the number of HSV infections and its severity has been reported. ${ }^{33}$ Also, numerous observational data suggest that HSV-2 genital infection facilitates HIV acquisition through genital tract mucosa. ${ }^{34,35}$ Therefore, it is interesting to test whether the Ag-NPs-coated PUC could also disrupt HSV infectivity. Indeed, the results presented in the study clearly showed that contact of the Ag-NPs-coated PUC to both HSV-1 and HSV-2 was able to efficiently inactivate their infectivity in Vero E6 cells (Figure 7). This indicates that, in addition to inhibiting HIV-1, the Ag-NPs-coated PUC is also efficient to prevent HSV-1/2 infection.

Of interest, the antibacterial and antifungal activity of the Ag-NPs-coated PUC was also observed in the study (Figure 8). It is believed that the antibacterial and antifungal activities of Ag-NPs are the result of the disruption of energy metabolism, electrolyte-transport systems, and/or distortion of bacterial cell wall. This disruption is due to the binding of Ag-NPs with bacterial sulphydryl- or histidyl-containing proteins. ${ }^{23,24}$ Recently, a study has shown that Ag-NPs arrested the cell cycle at the G2/M phase in C. albicans and also inhibited the budding process, probably through the destruction of membrane integrity. ${ }^{11}$ Obviously, more detailed investigation of the molecular mechanisms underlying the broad antibacterial and antifungal activity of the Ag-NPs-coated PUC will be necessary and will facilitate our better understanding of the broad actions of the Ag-NPs-coated PUC.

\section{Conclusions}

In conclusion, a method to coat the PUC with bioactive Ag-NPs was developed. These Ag-NPs bound to condoms 
are capable of inactivating a broad spectrum of microbial infectivity including that of HIV-1 and HSV-1/2. Moreover, since these bioactive Ag-NPs are tightly bound to the condom, and are easily removed with the condom after usage, it will significantly reduce the possibility of toxicity in chronic usage. This antimicrobial method will provide a safe and efficient way to disrupt different sexually transmitted infections, including HIV-1 and HSV infections, at the mucosal surface.

\section{Acknowledgments}

We would like to thank Drs Alberto Severini and Gary Kobinger for providing HSV-1/-2 virus strains and Vero-E6 cells. We also thank Dr M Emerman for the HeLa-CD4-CCR5- $\beta$-Gal cells that were obtained through the AIDS Research Reference Reagent Program, Division of AIDS, NIAID, NIH. We also greatly appreciate the editorial review of Ms Courtney Bell. Z-J Ao is the recipient of a postdoctoral fellowship from the CIHR International Infectious Disease and Global Health Training Program (IID and GHTP). This work was financially supported by grants from a CIHR HIV prevention operating grant (HPR85525), the Canadian Foundation for AIDS Research (CANFAR grant\# 023-013), and the Leaders Opportunity Fund Award from the Canadian Foundation of Innovation (CFI) to X-J Yao.

\section{Disclosure}

The authors report no conflicts of interest in this work.

\section{References}

1. UNAIDS. More than 80 countries increase their domestic investments for AIDS by over $50 \%$ between 2006 and 2011. July 18, 2012. Available from: http://www.unaids.org/en/resources/presscentre/pressreleaseandstatementarchive/2012/july/20120718prunaidsreport/. Accessed on August 12, 2012.

2. Pauwels R, DeClercq E. Development of vaginal microbicides for the prevention of heterosexual transmission of HIV. J Acquir Immune Defic Syndr Hum Retrovirol. 1996;11(3):211-221.

3. Balzarini J, Van Damme L. Microbicide drug candidates to prevent HIV infection. Lancet. 2007;369(9563):787-797.

4. Turpin JA. Considerations and development of topical microbicides to inhibit the sexual transmission of HIV. Expert Opin Investig Drugs. 2002;11(8):1077-1097.

5. Kippley J, Kippley S. The Art of Natural Family Planning. 4th ed. Cincinnati, OH: The Couple to Couple League; 1996.

6. Mamo T, Moseman EA, Kolishetti N, et al. Emerging nanotechnology approaches for HIV/AIDS treatment and prevention. Nanomedicine. 2010;5(2):269-285.

7. Porcel E, Liehn S, Remita H, et al. Platinum nanoparticles: A promising material for future cancer therapy? Nanotechnology. 2010;21(8): 085103.

8. Jain KK. Nanodiagnostics: Application of nanotechnology in molecular diagnostics. Exp Rev Mol Diagn. 2003;3(2):153-161.
9. Wang J, Wang L, Sun Y, et al. Surface plasmon resonance biosensor based on Au nanoparticle in titania sol-gel membrane. Colloids Surf B Biointerfaces. 2010;75(2):520-525.

10. Sondi I, Salopek-Sondi B. Silver nanoparticles as antimicrobial agent: A case study on E. coli as a model for Gram-negative bacteria. J Colloid Interface Sci. 2004;275(1):177-182.

11. Keuk-Jun K, Woo SS, Bo K, et al. Antifungal activity and mode of action of silver nano-particles on Candida albicans. Biometals. 2009; 22(2):235-242.

12. Lu L, Sun RW, Chen R, et al. Silver nanoparticles inhibit hepatitis B virus replication. Antivir Ther. 2008;13(2):253-262.

13. Sun RW, Chen R, Chung NP, Ho CM, Lin CL, Che CM. Silver nanoparticles fabricated in Hepes buffer exhibit cytoprotective activities toward HIV-1 infected cells. Chem Commun. 2005;28(40):5059-5061.

14. Elechiguerra JL, Burt JL, Morones JR, et al. Interaction of silver nanoparticles with HIV-1. J Nanobiotechnology. 2005;3(6):3-6.

15. Lara HH, Ayala-Nunez NV, Ixtepan-Turrent L, Rodriguez-Padilla C. Mode of antiviral action of silver nanoparticles against HIV-1. J Nanobiotechnology. 2010;8(1):1-8.

16. Jain P, Pradeep T. Potential of silver nanoparticle-coated polyurethane foam as an antibacterial water filter. Biotech. and Bioeng. 2005;90(1): $59-63$.

17. Ao Z, Yu Z, Wang L, Zheng Y, Yao X. Vpr14-88-Apobec3G fusion protein is efficiently incorporated into Vif-positive HIV-1 particles and inhibits viral infection. PLoS One. 2008;3(4):1995.

18. Zahariadis G, Severini A. Evaluation of a novel serology algorithm to detect herpes simplex virus 1 or 2 antibodies. Sex Transm Dis. 2010; 37(4):696-699.

19. Burleson FG, Chambers TM, Wiedbrauk DL. Plaque assay. In: Burleson FG, Chambers TM, Wiedbrauk DL, editors. Virology: A Laboratory Manual. San Diego, CA: Academic Press; 1992:74-84.

20. Kimpton J, Emerman M. Detection of replication-competent and pseudotyped human immunodeficiency virus with a sensitive cell line on the basis of activation of an integrated b-galactosidase gene. JVirol. 1992;66(4):2232-2239.

21. Ao Z, Fowke KR, Cohen EA, et al. Contribution of the C-terminal tri-lysine regions of HIV-1 integrase for efficient reverse transcription and viral DNA nuclear import. Retrovirology. 2005, 2(62):1-15.

22. Fayaz AM, Balaji K, Girilal M, Ruchi Y, Kalaichelvan PT, Venkatasen R. Biogenic synthesis of silver nanoparticles and their synergistic effect with antibiotics: a study against Gram-positive and Gram-negative bacteria. Nanomedicine. 2010;6(1):103-109.

23. Guggenbichler JP, Boswald M, Lugauer S, Krall T. A new technology of microdispersed silver in polyurethane induces antimicrobial activity in central venous catheters. Infection. 1999;22(1):16-23.

24. Centers for Disease Control and Prevention. Condoms and STDs: Fact sheet for public health personnel. Atlanta, GA: Center for Disease Control and Prevention. [Updated September 13, 2011; cited November 12,

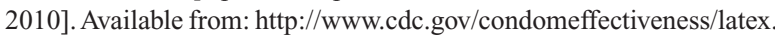
htm. Accessed October 17, 2011.

25. Handley MA, Reingold AL, Shiboski S, Padian NS. Incidence of acute urinary tract infection in young women and use of male condoms with and without nonoxynol-9 spermicides. Epidemiology. 2002;13(4):431-436.

26. World Health Organization. Nonoxynol-9 ineffective in preventing HIV infection. [Cited Jul 2006] Available from: http://www.who.int/mediacentre/news/notes/release55/en/index.html. Accessed July 10, 2009.

27. Bhol KC, Schechter PJ. Tropical nanocrystalline silver cream suppresses inflammatory cytokines and induces apoptosis of inflammatory cells in a murine model of allergic contact dermatitis. Br J Dermatol. 2005; 152(6):1235-1242.

28. Sibbald RG, Contreras-Ruiz J, Coutts P, Fierheller M, Rothaman A, Woo K. Bacteriology inflammation and healing a study of nanocrystalline silver dressing in chronic venous leg ulcers. Adv Skin Wound Care. 2007;20(10):549-558.

29. Baker C, Pradhan A, Pakstis L, Pochan DJ, Shah SI. Synthesis and antibacterial properties of silver nanoparticles. J Nanosci Nanotechnol. 2005;5(2):244-249. 
30. Lara HH, Ixtepan-Turrent L, Garza-Trevino EN, Rodriguez-Padilla C. PVP-coated silver nanoparticles block the transmission of cell-free and cell-associated HIV-1 in human cervical culture. J Nanobiotechnology. 2010;8(15):8-15.

31. Rai M, Yadav A, Gade A. Silver nanoparticles as a new generation of antimicrobials. Biotech Adv. 2009;27(1):76-83.

32. Spira S, Wainberg MA, Loemba H, Turner D, Brenner BG. Impact of clade diversity on HIV-1 virulence, antiretroviral drug sensitivity and drug resistance. J Antimicrob Chemother. 2003;51(2):229-240.
33. Cassidy KA, Whitley RJ. New therapeutic approaches to the alpha herpes virus infections. J Antimicrob Chemother. 1997;39(2):119-128.

34. Barnabas RV, Laukkanen P, Koskela P, Kontula O, Lehtinen M, Garnett PG. Epidemiology of HPV 16 and cervical cancer in Finland and the potential impact of vaccination: mathematical modelling analyses. PLoS Med. 2006;3(5):e138.

35. Weiss HA, Buvé A, Robinson NJ, et al. The epidemiology of HSV-2 infection and its association with HIV infection in four urban African populations. AIDS. 2001;15:S97-S108.

\section{Publish your work in this journal}

The International Journal of Nanomedicine is an international, peerreviewed journal focusing on the application of nanotechnology in diagnostics, therapeutics, and drug delivery systems throughout the biomedical field. This journal is indexed on PubMed Central, MedLine, CAS, SciSearch $\AA$, Current Contents ${ }^{\circledR} /$ Clinical Medicine,
Journal Citation Reports/Science Edition, EMBase, Scopus and the Elsevier Bibliographic databases. The manuscript management system is completely online and includes a very quick and fair peer-review system, which is all easy to use. Visit http://www.dovepress.com/ testimonials.php to read real quotes from published authors. 